\title{
Optimization of a Fabry-Perot cavity operated in burst mode for Compton scattering experiments
}

\author{
Pierre Favier, Loïc Amoudry, Kevin Cassou, Ronic Chiche, Kevin Dupraz, Aurélien Martens," \\ Daniele Nutarelli, Viktor Soskov, and Fabian Zomer \\ LAL, Univ. Paris-Sud, CNRS/IN2P3, Université Paris-Saclay, 91898 Orsay, France \\ Antoine Courjaud \\ Amplitude Systèmes, 11, Avenue de Canteranne, Cité de la Photonique, 33600 Pessac, France \\ Luca Serafini \\ INFN-MI, Via Celoria 16, 20133 Milano, Italy
}

(Received 20 September 2018; published 12 December 2018)

\begin{abstract}
Formal and experimental studies of Fabry-Perot cavities operating in burst mode are conducted in the context of Compton scattering. A dedicated optimization procedure for their use in this context is described for the first time, to the best of our knowledge. Provided that the number of bunches in the electron-beam train and their time separation are known, the parameters related to the optical system are chosen to maximize the ratio of the total intracavity laser-beam energy used for collisions to the total input laser beam seeded into the cavity operated in burst mode. A good qualitative agreement is obtained between the model and the experimental data. It is shown by studying the upgrade of an existing machine, STAR. Here a new optical system with increased performance without a large increase in complexity is obtained. This result paves the way towards future designs of high-flux, high-quality, low cost and compact x-ray facilities based on Compton scattering.
\end{abstract}

DOI: 10.1103/PhysRevAccelBeams.21.121601

\section{INTRODUCTION}

Fabry-Perot cavities (FPCs) [1] in the pulsed regime have high-power applications in high harmonic generation [2,3], polarized positron generation [4], and $\mathrm{x}$ - or $\gamma$-ray production by Compton scattering [5-18]. In this article, we focus on the latter application. Most Compton sources are made either of a linear accelerator (linac), coupled to an optical circulator [5-7] or a single laser pulse; or a storage ring to a FPC [8-12]. However, for operation in small areas, e.g. inside hospitals, museums or university labs, such machines must shrink to their simplest elements to reduce cost and footprint $[12,13]$. In order to preserve the excellent x-ray beam spectral linewidth related to the excellent e-beam quality achievable in linacs [5] and to maximize the produced x-ray flux, a high power FPC may be coupled to a room-temperature linac $[19,20]$. Due to peak-power constraints in klystrons used to feed rf cavities, linacs can only provide e-beams with few microsecond train duration

\footnotetext{
*martens@1al.in2p3.fr
}

Published by the American Physical Society under the terms of the Creative Commons Attribution 4.0 International license. Further distribution of this work must maintain attribution to the author(s) and the published article's title, journal citation, and DOI. at macrorepetition rates of at most few hundreds of hertz. The FPC can then be operated in permanent regime or in burst mode [20-22] for which the filling cycle is matched to that of the electron beam duration. The peak power, of interest for collisions with electrons, can in principle be higher in burst mode than in permanent regime, provided that an optimization is performed.

Burst mode cavities have been used for hydrogen ion stripping [23] and multistage laser plasma accelerators $[24,25]$. They are seeded by bursts of laser pulses, leading to a decrease by several orders of magnitude of the storage time compared to the permanent regime operation. As a result, a very high input-beam energy can be injected at low duty cycle without inducing significant thermal deformations on the mirrors [26] owing to the lower average power inside the cavity. In this regime, the performances are thus limited by the damage threshold of the cavity mirrors [27]. Burst mode FPCs have already been used in Compton experiments $[20,21]$. To the best of our knowledge, no systematic optimization of the laser and electron beam parameters has been done so far prior to operation. It aims at maximizing the Compton backscattered photon flux.

In this article, the existence of optimum conditions is demonstrated both numerically and experimentally. These conditions are related to the finesse of the FPC, the arrival time of the first electron bunch, and the duration and energy 
of the input laser-beam pulse train. This article is organized as follows. Formal expressions used to optimize the parameters of the burst operation are presented in Sec. II. The benefits of the optimization are demonstrated in Sec. III on a study case which consists in the upgrade of an existing machine. Details of the experimental setup used to validate the optimization procedure of the FPC operated in burst mode are given in Sec. IV.

\section{FORMALISM}

A laser beam composed of $N_{p}$ laser pulses, as represented on Fig. 1 (top), is injected into a FPC. The energy of the pulse stacked in the cavity increases after each round-trip. It decreases when the $N_{p}$ incident laser pulses have all been injected, see Fig. 1 (middle). After the injection of $N_{0}$ laser pulses, a train of $N_{e}$ electron bunches interacts with the laser pulse stored in the FPC, see Fig. 1 (bottom). It is assumed that the laser pulses and electron bunches time separation are identical, that is the FPC and laser frequencies are locked on the rf clock. In addition, all the laser pulses have the same initial energy $\mathcal{E}_{0}$, and all the electron bunches have the same charge $Q$. The effect of an energy spread over the electron bunch train in the x-ray spectral line due to beam loading effects in the linac is not discussed in this paper. It will be the subject of a future work, as it mainly affects the bandwidth of the emitted radiation. Mitigation of energy spread broadening due to beam loading does exist, see for instance $[28,29]$. The total laser energy available for the Compton interaction is denoted $\mathcal{E}_{\text {tot }}$. It corresponds to the sum of the energies of the laser pulses that interact with electron bunches. The output $\mathrm{x}$-ray flux is thus

$$
\frac{d n_{X}}{d t}=\sigma_{C} \frac{Q N_{b} \mathcal{E}_{\text {tot }} f_{\text {rep }}}{2 \pi e h c / \lambda} \Upsilon
$$

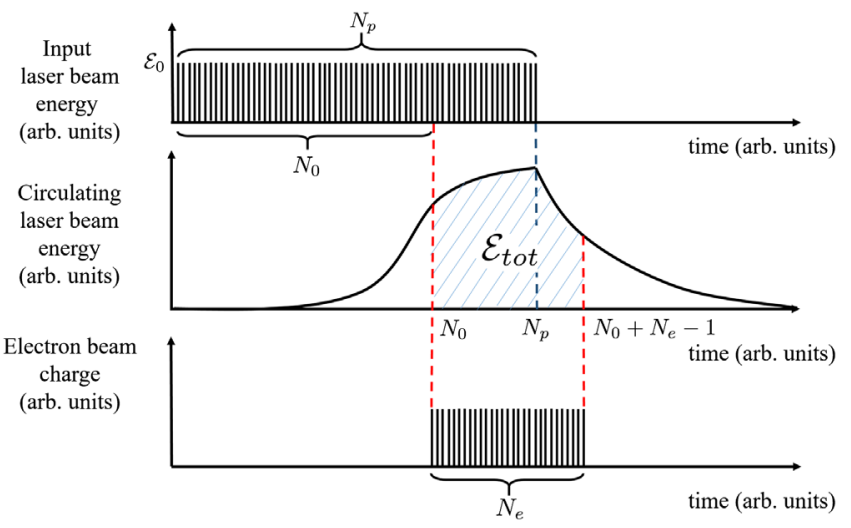

FIG. 1. Temporal patterns of (top) the input laser beam pulse train energy, (middle) the intracavity laser beam energy and (bottom) the electron beam bunch train intensity, for the use of a burst mode FPC in Compton experiments. The total effective laser beam energy used for Compton scattering off the $N_{e}$ electron bunches is denoted $\mathcal{E}_{\text {tot }}$. where $\sigma_{C}$ is the total Compton cross section, $f_{\text {rep }}$ the electron train repetition frequency, $N_{b}$ is the number of electron bunches in the train, $e$ the elementary charge, $h$ the Planck's constant, $c$ the speed of light in vacuum, and $\lambda$ the laser beam wavelength. In practice, for most applications and in particular for that studied in Sec. III, $\sigma_{C} \simeq \sigma_{T} \simeq 0.665$ barn where $\sigma_{T}$ is the Thomson cross section. Assuming Gaussian functions for laser and electron beam distributions and neglecting their divergences [30],

$$
\Upsilon=\frac{1}{\sqrt{\sigma_{y e}^{2}+\sigma_{y \ell}^{2}} \sqrt{\left(\sigma_{x e}^{2}+\sigma_{x \ell}^{2}\right)+\left(\sigma_{z e}^{2}+\sigma_{z \ell}^{2}\right) \tan ^{2} \frac{\alpha}{2}}},
$$

where $\alpha$ is the crossing angle in the incidence $x$ - $z$ plane between the laser and electron beams and $\sigma_{a b}$ is the Gaussian standard deviation along the direction $a \in\{x, y, z\}$ for the electron $(b=e)$ and laser $(b=\ell)$ beam.

The effective energy $\mathcal{E}_{\text {tot }}$ is a function of $\mathcal{E}_{0}, N_{0}, N_{p}, N_{e}$, and the cavity finesse $F$. It reads

$$
\mathcal{E}_{\text {tot }}=\epsilon\left(N_{p}, N_{0}, N_{e}, F\right) N_{p} \mathcal{E}_{0},
$$

where the product $N_{p} \mathcal{E}_{0}$ is the total incident energy delivered to the cavity by the input laser amplifier, and $\epsilon$ is the effective gain of the optical system.

In the permanent regime $\left(N_{p} \rightarrow \infty\right)$, in which case $\epsilon=N_{e} T_{1} /\left[(1-r)^{2} N_{p}\right]$, with $T_{1} /(1-r)^{2}$ being the enhancement factor of the FPC, $T_{1}$ the cavity input mirror transmission coefficient in power, and $r=r_{1} r_{2} \ldots r_{n}$ the product of the reflection coefficients in the field of the set of $n$ mirrors forming the FPC. In this paper, the losses related to the Compton interaction itself are negligible, and thus do not appear in the definition of enhancement factor of the FPC. This configuration induces limitations on the input laser amplifier that must run continuously at frequencies of the order of a few hundreds of $\mathrm{MHz}$ leading to an energy per pulse $\mathcal{E}_{0} \simeq 1 \mu \mathrm{J}$ for the state-of-the-art fiber amplifiers [26]. This system typically leads to a total available energy in the cavity $\mathcal{E}_{\text {tot }}$ of about one joule for $N_{e}=100$ and $F=$ 30000 which corresponds to $\epsilon=1$ assuming that the repetition rate of the train of electron bunches is of $100 \mathrm{~Hz}$. It would lead to one megawatt of stored average power which is beyond the current state of the art [26] and may lead to instabilities [31]. In burst mode however we will show that an optimum set of parameters that maximize $\epsilon$ and hence the x-ray flux exists. In such a configuration, solid-state laser amplifiers are available and allow the extraction of several tens of watts at several hundreds of hertz $[32,33]$. This kind of amplifiers can be seeded by bursts of laser-beam pulses and are suited for the case studied in this article. As will be seen in Sec. III, this configuration leads to $\epsilon \simeq 100-1000$, which also leads to about joule-level stacked total energy. In addition, the low repetition frequency of the laser burst would significantly 
decrease the average power stored in the cavity that leads to modal instabilities induced by the mirror coating thermal load [26,31]. The remainder of this article is dedicated to the optimization of this second solution.

According to Fig. 1, the effective energy

$$
\mathcal{E}_{\text {tot }}=\sum_{p=N_{0}}^{N_{p}} \mathcal{E}_{p}^{\text {fill }}+\sum_{p=N_{p}+1}^{N_{0}+N_{e}-1} \mathcal{E}_{p-N_{p}}^{\text {decay }}
$$

available for the interaction with the $N_{e}$ electron bunches is the sum of the stored energy between $N_{0}$ and $N_{0}+N_{e}-1$. On the one hand, it makes no sense to send optical pulses in the cavity once the last electron bunch has passed. On the other hand, sending electrons only during the cavity decay process makes no use of the maximum energy available which occurs when $N_{p}$ laser pulses have been injected. The optimum set of parameters should thus lie in the range $N_{0} \leq N_{p} \leq N_{0}+N_{e}-1$.

The energy stacked in the FPC during filling time and after $p$ laser pulses have been injected reads

$\mathcal{E}_{p}^{\text {fill }}=\int_{-\infty}^{+\infty}\left|\sqrt{T_{1}} \sum_{n=0}^{p-1} r^{n} E_{n}(t)\right|^{2} d t=\mathcal{E}_{0} T_{1}\left(\frac{1-r^{p}}{1-r}\right)^{2}$,

where $\mathcal{E}_{0}=\int_{-\infty}^{+\infty}\left|E_{n}(t)\right|^{2} d t \forall n$ is the energy in a single input laser pulse, and $E_{n}(t)$ is the electric field of the $n$th laser pulse taken at time $t$. After $N_{p}$ laser pulses have been injected in the FPC, the stored energy flows out. The stored pulse energy reads

$$
\mathcal{E}_{q}^{\text {decay }}=\mathcal{E}_{N_{p}}^{\text {fill }} r^{2 q}
$$

$q$ cavity round-trips after that time. From Eqs. (4)-(6), one obtains

$$
\begin{aligned}
\epsilon= & \frac{T_{1}}{(1-r)^{2}}\left(1-\frac{N_{0}}{N_{p}}+\frac{2 r\left(r^{N_{p}}-r^{N_{0}}\right)}{N_{p}\left(1-r^{2}\right)}\right. \\
& \left.+\frac{\left(1-r^{N_{0}}\right)^{2}-r^{2 N_{e}} r^{2 N_{0}}\left(1-r^{-N_{p}}\right)^{2}}{N_{p}\left(1-r^{2}\right)}\right) .
\end{aligned}
$$

From this equation it is clear that for a given finesse value $F$, i.e. a given value of $r$, and given $N_{e}, N_{p}$ and $N_{0}$ values, it is of interest to maximize $T_{1}$, i.e. to work with an overcoupled cavity. This is the case if the total losses of cavity do not exceed $\pi / F$. It can be checked that the optimum values found in this paper always verify this assumption. For a given value of $N_{e}$, it appears that there exists values of $N_{p}, N_{0}$ and $T_{1}$ that maximize $\epsilon$. To show this one also specifies a value of $N_{p}$ and searches for solutions of the set of equations $\left\{\partial \epsilon / \partial T_{1}=0, \partial \epsilon / \partial N_{0}=0\right\}$. The cavity finesse is assumed here to be only driven by $T_{1}$, i.e. the reflectivity of each other mirror $r_{i>1} \equiv r_{2}, \ldots, r_{n}$ is equal to $r_{\max }^{2} \simeq 99.999 \%$ [34]. Solving $\partial \epsilon / \partial N_{0}=0$ gives a formal solution

$$
N_{0}^{\mathrm{opt}}=\frac{1}{\ln (r)} \ln \left(\frac{1}{2 R \ln (r)}[1-\sqrt{1+2 R(1-r)}]\right)
$$

with

$$
R=\frac{r^{2 N_{p}}-r^{2 N_{e}}\left(1-r^{N_{p}}\right)^{2}}{r^{2 N_{p}}(1+r) \ln r}
$$

which holds in the range $N_{p} \in\left[N_{0}, N_{0}+N_{e}-1\right]$. Injecting $N_{0}=N_{0}^{\text {opt }}$ into Eq. (7), the solution $T_{1}^{\text {opt }}$ of the equation $\left\{\partial \epsilon / \partial T_{1}=0\right\}_{N_{0}=N_{0}^{\text {opt }}}$ is solved numerically. Finally the value of $N_{p}$ is numerically optimized solving

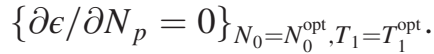

Simpler expressions are obtained by taking the continuous filling time limit [35]. For a FPC of finesse greater or around few hundreds, as those considered in this article, the round-trip time $T_{c}$ is much smaller than the photon typical lifetime $T_{\text {life }} \simeq F T_{c} /(2 \pi)$. Previous equations can then be approximated by converting the number of pulses into time intervals $p \rightarrow t / T_{c}$. $N_{p}$ then is replaced by $t_{p} / T_{c}, N_{e}$ by $t_{e} / T_{c}$, and $N_{0}$ by $t_{0} / T_{c}$. The effective gain thus becomes

$$
\begin{aligned}
\epsilon= & \frac{T_{1}}{(1-r)^{2}} \frac{1}{x}\left[x+2 e^{-x / 2}-\left(z+2 e^{-z / 2}\right)\right. \\
& \left.+\left(1-e^{-z / 2}\right)^{2}-e^{-z-y}\left(1-e^{x / 2}\right)^{2}\right],
\end{aligned}
$$

with $x=2 t_{p} / \tau, y=2 t_{e} / \tau, z=2 t_{0} / \tau$ and $\tau=-T_{c} / \ln r$ the time constant of the cavity filling process. By solving the equation $\partial \epsilon / \partial t_{0}=0$, we also find the optimum value for $t_{0}$ :

$$
t_{0}^{\mathrm{opt}}=\tau \ln \left[1-e^{-y / 2}\left|1-e^{x / 2}\right|\right]
$$

\section{NUMERICAL STUDIES}

In order to show the performances that may be reached by using an optimized burst mode cavity, a realistic case is considered. Electron beam parameters of the STAR project [36,37] are used for that purpose. A 30-95 MeV electron bunch with a charge of $500 \mathrm{pC}$, normalized emittance of $0.9 \mathrm{~mm} \cdot \mathrm{mrad}$ and $0.2 \%$ energy spread will collide with a $400 \mathrm{~mJ}$ laser pulse at a frequency of $100 \mathrm{~Hz}$. The laser beam central wavelength is $1030 \mathrm{~nm}$. Each electron bunch is expected to have a longitudinal Gaussian shape with $\sigma=$ $3.5 \mathrm{ps}$ and the laser pulses duration is $5 \mathrm{ps}$ FWHM. At the Compton interaction point the laser and electron beams spot sizes are $20 \times 20$ and $(15 \times 15) \mu \mathrm{m}^{2}$, respectively. The laser-electron beams crossing angle is 2.3 degrees. The $\mathrm{X}$-ray yield is $n_{\mathrm{X}} \approx 6 \times 10^{9} \mathrm{~s}^{-1}$ with these parameters [37]. 
TABLE I. Optimized burst mode parameters for the STAR parameters. See text for details.

\begin{tabular}{lrrrrrc}
\hline \hline$N_{e}$ & \multicolumn{1}{c}{$N_{p}$} & \multicolumn{1}{c}{$N_{0}$} & \multicolumn{1}{c}{$\epsilon$} & \multicolumn{1}{c}{$F$} & $G_{\max }$ & $\bar{G} /\left(N_{e} G_{\max }\right)$ \\
\hline 250 & 2805 & 2625 & 190 & 6642 & 2210 & 0.96 \\
1000 & 5773 & 5043 & 714 & 12926 & 4392 & 0.94 \\
250 & 250 & 115 & 132 & 697 & 202 & 0.65 \\
1000 & 1000 & 455 & 525 & 2745 & 801 & 0.65 \\
\hline \hline
\end{tabular}

In STAR the rf pulse duration cannot exceed $1 \mu \mathrm{s}$. We consider the two following schemes for a burst version of STAR: $N_{e}=250\left(T_{c}=250 \mathrm{MHz}^{-1}=4 \mathrm{~ns}\right)$ and $N_{e}=$ $1000\left(T_{c}=1 \mathrm{GHz}^{-1}=1 \mathrm{~ns}\right)$. From Eq. (7) we obtain the effective gain $\epsilon$ and $N_{p}$ from its numerical maximization. Results are shown in Table I where the input value of $N_{e}$ is given along with the values of $N_{p}, N_{0}, \epsilon, F$, the maximum gain reached in the cavity $G_{\max }=\max \left(\mathcal{E}_{p}\right) / \mathcal{E}_{0}$ and the ratio $\bar{G} /\left(N_{\mathrm{e}} G_{\max }\right)=\mathcal{E}_{\text {tot }} /\left(N_{e} \max \left(\mathcal{E}_{p}\right)\right)$ which quantifies the gain flatness of the burst mode cavity during the collision time. It is found that $N_{p}$ is significantly higher than $N_{e}$. In this case the gain is nearly flat in the burst mode FPC, a 5\% loss of flux compared to a continuously filled cavity is thus expected. Depending on the laser burst amplifier architecture it may also be useful to reduce $N_{p}$. The two last lines of Table I correspond to the case where we arbitrarily fix $N_{e}=N_{p}$. Though there is an optimum in the burst cavity parameters, the range which provides solutions close to the optimum is rather large. The optimum solutions and the contour plots of the areas for which $\epsilon>0.99 \max (\epsilon)$ and $\epsilon>0.90 \max (\epsilon)$ are shown in Figs. 2 and 3. Interestingly, the case $N_{e}=N_{p}$ is clearly excluded from the region of interest.

To quantify the actual photon flux, and the maximum allowed laser pulse energy inside the cavity, one has to specify the burst cavity geometry. A symmetric four-mirror geometry made of two planar $\left(\mathrm{M}_{1}\right.$ and $\mathrm{M}_{2}$ in Fig. 4) and two spherical mirrors ( $\mathrm{M}_{3}$ and $\mathrm{M}_{4}$ in Fig. 4) of radius of curvature $R$ is assumed. The mirror diameters are denoted $\Phi$ and it is assumed that high reflective coating covers $80 \%$ of the mirror surface, i.e. a standard of high quality polishing and coating. One assumes that $\mathcal{F}_{\max }=2 \mathrm{~J} / \mathrm{cm}^{2}$ for the laser damage threshold at $1030 \mathrm{~nm}$ [27]. The optical cavity round-trip length $L_{\mathrm{RT}}=1.2$ or $0.3 \mathrm{~m}$ is assumed to be matched to the repetition rate of the laser; i.e. it is not operated on a (sub)harmonic of the repetition rate. The optimization is performed for a $E_{e}=30 \mathrm{MeV}$ electron beam, which corresponds to an angular distribution of the radiation that is more extended transversely compared to higher electron beam energies available at STAR. The minimal acceptable crossing angle is thus assumed to be $\alpha=1 / \gamma+\Phi /\left(2 d_{1}\right)$. The length $d_{1}$ is calculated as $d_{1}=\left(L_{\mathrm{RT}}^{2}-4 L_{\mathrm{RT}} d_{2}-4 h^{2}\right) /\left(4 L_{\mathrm{RT}}\right)$. Knowing the two geometrical parameters $h$ and $d_{2}$ and the $R$ value, the optical beam size at the waist and on the four mirrors is

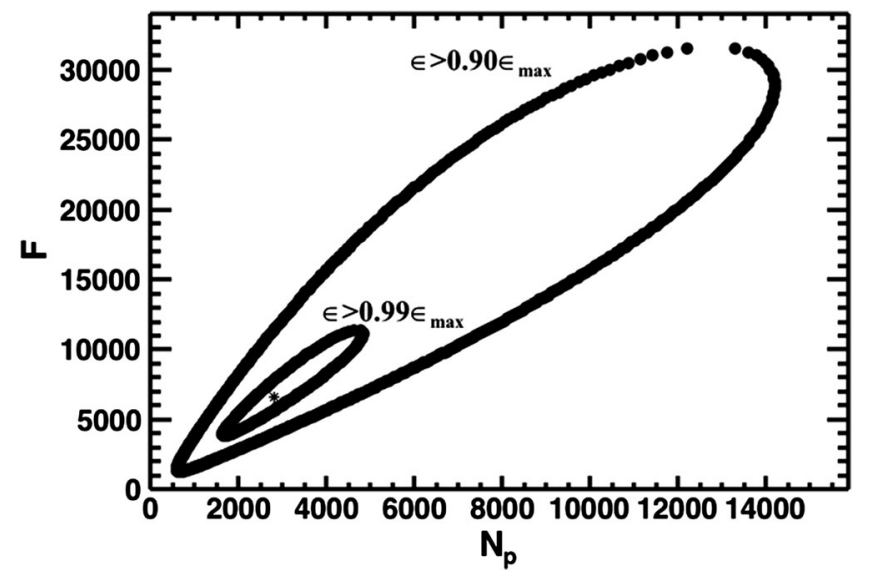

FIG. 2. Optimum values for $F$ and $N_{p}$ and contour plots delimiting areas where $\epsilon>0.90 \epsilon_{\max }$ and $\epsilon>0.99 \epsilon_{\max }$ for $N_{e}=250$.

computed by ABCD matrix formalism [38]. The parameters $h, d_{2}$, and $R$ are taken randomly in the range $\left[0, L_{\mathrm{RT}} / 4\right]$ and the laser pulse energy $U$ is randomized within a reasonable energy range. Three constraints are imposed on these Monte Carlo trials to reject unacceptable solutions: (i) the optical beam impinging the mirrors is constrained not to be eclipsed by another mirror which turns into a nonlinear (since $d_{1}$ depends in turn on $h$ ) constraint $h-\Phi>h\left|d_{1}-d_{2}\right| /\left(d_{1}+d_{2}\right)$; (ii) the fluence $\mathcal{F}=2 U /\left(\pi w_{s} w_{t}\right)$ is assumed to be less than $\mathcal{F}_{\text {max }}$ both on plane and spherical mirrors; (iii) the absolute value of the trace of the $\mathrm{ABCD}$ matrices in sagittal and tangential planes are constrained to be smaller than 2 (stable optical cavity).

This Monte Carlo technique proved to be very fast and efficient in scanning the whole parameter space to quickly provide viable designs. The solutions maximizing the flux are summarized in Table II for the two assumed cavity lengths. Due to the randomization technique these solutions are not absolute maxima but can be considered as solutions

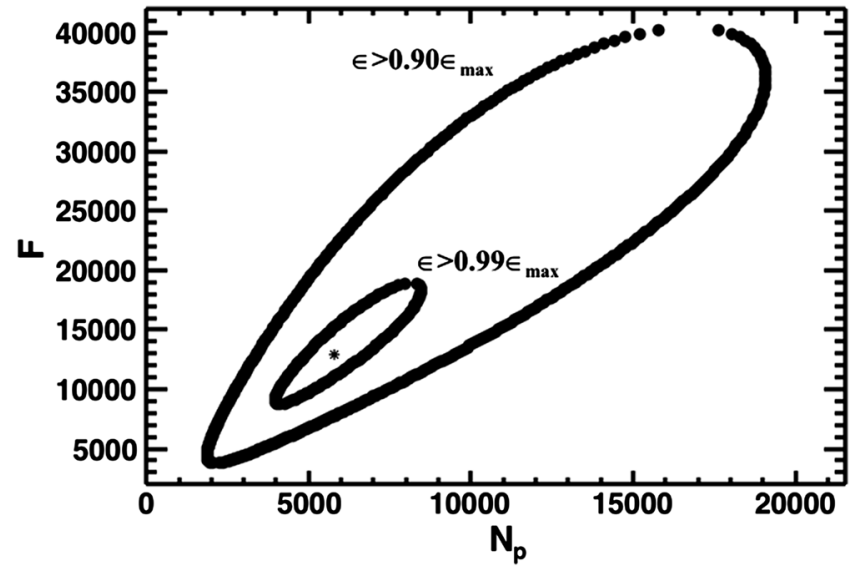

FIG. 3. Optimum values for $F$ and $N_{p}$ and contour plots delimiting areas where $\epsilon>0.90 \epsilon_{\max }$ and $\epsilon>0.99 \epsilon_{\max }$ for $N_{e}=1000$. 


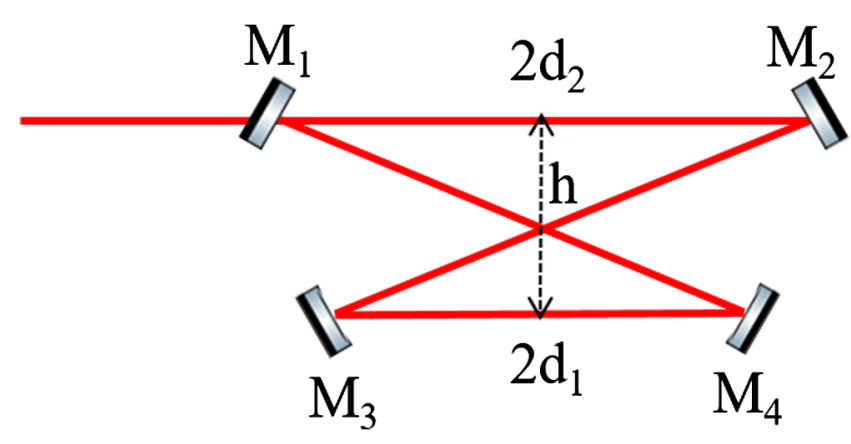

FIG. 4. Schematic view of a symmetric four-mirror planar cavity. $\mathrm{M}_{1}, \mathrm{M}_{2}\left(\mathrm{M}_{3}, \mathrm{M}_{4}\right)$ are planar (spherical) mirrors. Distances $d_{1}, d_{2}$ and $\mathrm{h}$ describing the geometry are also shown. Mirror positions are symmetric with respect to the central vertical axis.

very close to the optimum. The quoted flux in this table assumes that the pulse energy is constant in the cavity throughout the interaction of the electron bunches. This assumption is nearly correct when $N_{e} \ll N_{p}$ but breaks when $N_{e} \simeq N_{p}$, see the value of $\bar{G} /\left(N_{e} G_{\max }\right)$ in Table I.

Besides fixing the geometrical parameters, the maximum allowed energy in the cavity is thus fixed which in turn allows to determine the requirements on the laser system, namely the laser burst energy at the entrance of the optical cavity and the single pulse energy in the input burst laser. These parameters are listed in Table III along with the duration $T_{b}$ of the laser burst amplification. The optimization at $1 \mathrm{GHz}$ is less demanding on the IP laser system both in terms of burst duration and energy while at $250 \mathrm{MHz}$ a more powerful laser system is required. Such laser systems seem to be achievable in terms of pulse energy and average power requirements, looking at existing systems $[39,40]$.

The same laser system may be used for both IP and UV lasers. Indeed, using a CsTe cathode and assuming $1 \%$ quantum efficiency $[41,42]$ the required energy in the UV per pulse for the photocathode laser system is $0.24 \mu \mathrm{J}$. Assuming 10\% fourth-harmonic conversion efficiency, it corresponds to $2.4 \mu \mathrm{J} /$ pulse in the IR for the photocathode laser system.

Though the $1 \mathrm{GHz}$ solution seems more elegant, laser system wise, difficulties are translated to the electron beam. Beam-breakup instabilities will be large when dealing with 1000 bunches separated by 1 ns with 500 pC each which could constrain drastically this solution. Once a laser system architecture is drawn, a full optimization involving more detailed constraints on both laser and e-beam system could be accounted for to determine more precisely the design parameters of the optical cavity operated in burst mode. This further optimization is out of the scope of this paper.

\section{EXPERIMENTAL STUDY}

The optimization procedure of the FPC operated in burst mode is experimentally validated only for the optical part since we only intend to optimize the optical parameters. The experimental setup is depicted on Fig. 5. A continuous wave (cw) laser oscillator seeds a laser beam of $100 \mathrm{~mW}$ at $1030 \mathrm{~nm}$ to a fibered phase modulator (PM) used for lasercavity locking [43]. A fibered frequency shifter (FS1) is used to correct high-frequency noise in the feedback loop. The laser beam is split in two arms [21,44] using an optical fiber divider/combiner. The upper line, denoted locking line on Fig. 5, is used to permanently lock the oscillator on the FPC. On the lower line, denoted burst line on Fig. 5, an additional electro-optic modulator (EOM) driven by a ramp voltage is used as a frequency shifter (FS2). It is needed since $s$ - and $p$-waves resonate at different frequencies in the cavity, as explained further in the text. A polarizing beam splitter (PBS1) and a Faraday rotator (FR) isolate the oscillator from backward reflections. The laser beam is reflected off PBS2 and sent through a pulse picker (PP). The pulse picker is a Pockels cell used as a quarter wave plate. The Pockels cell is composed of two BBO crystals. The high voltage signal is driven by a square waveform of adjustable duration and frequency. When no high voltage is applied on the Pockels cell, the laser beam goes backwards through the isolator and the signal is monitored with a photodiode (PD1). When the high voltage is applied, the laser beam is transmitted through PBS2 and injected in the FPC. The lock and burst lines are put on different polarization states using half and quarter wave plates. A nonpolarizing beam splitter (BS) is used to combine them before injection. The signal reflected from the FPC is filtered in polarization and only the contribution from the locking line is sent to a photodiode PD4 and a servo for Pound-Drever-Hall (PDH) locking. An avalanche photodiode (APD) is used to trigger the feedback system on the $\mathrm{TEM}_{00}$ mode of the FPC. The photodiodes PD2 and PD3 are used to monitor the transmissions of the locking and burst lines respectively.

The FPC is composed of four mirrors in a planar configuration located inside a vacuum vessel. The coatings of the cavity mirrors have been adjusted to provide round-trip phase detuning between $s$ - and $p$-waves at the nominal incidence angle, leading to two different resonance frequencies [45]. This frequency difference is approximately $20 \mathrm{kHz}$ and is

TABLE II. Optimized geometries of the burst mode cavity for two different values of $L_{\mathrm{RT}}$. See text for details.

\begin{tabular}{lccccccccc}
\hline \hline$L_{\mathrm{RT}}[\mathrm{m}]$ & $2 d_{1}[\mathrm{~cm}]$ & $2 d_{2}[\mathrm{~cm}]$ & $h[\mathrm{~cm}]$ & $\alpha$ [degree] & $R[\mathrm{~cm}]$ & $\Phi[\mathrm{cm}]$ & $U_{\max }[\mathrm{mJ}]$ & $w_{0 s} / w_{0 t}[\mu \mathrm{m} / \mu \mathrm{m}]$ & $n_{X}$ [photons/s] \\
\hline 1.2 & 24.4 & 35.5 & 1.66 & 4.1 & 24.4 & 1.35 & 130 & $14 / 26$ & $6.9 \times 10^{11}$ \\
0.3 & 6.6 & 8.31 & 0.77 & 6.0 & 6.6 & 0.587 & 22 & $9 / 19$ & $3.6 \times 10^{11}$ \\
\hline \hline
\end{tabular}


TABLE III. Optimized parameters for the input laser beam for the STAR configuration. $P_{\text {peak }}$ denotes the ratio of the burst energy $E_{\text {burst }}$ and the burst duration $T_{b}$, while $P_{\text {peak,cavity }}$ is the laser power stored in the cavity during collisions with electrons.

\begin{tabular}{lcccccc}
\hline \hline$N_{e}$ & $N_{p}$ & $T_{b}$ & $E_{\text {burst }}$ & $E_{\text {pulse }}$ & $P_{\text {average }}$ & $P_{\text {peak }}$ \\
\hline 250 & 2805 & $11 \mu \mathrm{s}$ & $165 \mathrm{~mJ}$ & $59 \mu \mathrm{J}$ & $16.5 \mathrm{~W}$ & $15 \mathrm{~kW}$ \\
1000 & 5773 & $5.8 \mu \mathrm{s}$ & $29 \mathrm{~mJ}$ & $5 \mu \mathrm{J}$ & $2.9 \mathrm{~W}$ & $5 \mathrm{~kW}$ \\
250 & 250 & $1 \mu \mathrm{s}$ & $160 \mathrm{~mJ}$ & $640 \mu \mathrm{J}$ & $16.0 \mathrm{~W}$ & $160 \mathrm{~kW}$ \\
1000 & 1000 & $1 \mu \mathrm{s}$ & $27 \mathrm{~mJ}$ & $27 \mu \mathrm{J}$ & $2.7 \mathrm{~W}$ & $21 \mathrm{MW}$ \\
\hline \hline
\end{tabular}

greater than the cavity frequency linewidth. Therefore, a frequency detuning of $20 \mathrm{kHz}$ between lock and burst laser beam lines must be provided to lock them simultaneously. This is the purpose of the EOM denoted FS2 in Fig. 5. This EOM provides a linear phase modulation, whose slope defines the constant frequency shift. This frequency shift is adjusted by changing the voltage and the period of the ramp. Due to the sharp voltage variation at the end of the ramp, this technique adds a phase jump in the signal. From experimental observations and calculations, this phase jump was found to have a negligible impact on the results.

Data are recorded on the APD. To cancel the visible $20 \mathrm{kHz}$ frequency modulation, the Pockels cell driving square waveform is not synchronized with the ramping phase modulation, and the signal is averaged 512 times on the oscilloscope. Data are baseline corrected and normalized by the cavity steady-state voltage value. Data have been taken from $t_{p}=5 \mu \mathrm{s}$ to $150 \mu$ s by steps of $2.8 \mu \mathrm{s}$. An example corresponding to $t_{p}=100 \mu$ s is shown on Fig. 6 with the corresponding Pockels cell opening duration as measured from PD1.

No attempt was made to calibrate the APD, thus APD response was normalized by its asymptotic value. Experimental relative effective gains are thus derived from data by integrating numerically the APD response varying the starting time and the duration of the integration. Experimental effective gains $\epsilon$ are calculated as a function of $t_{p}$ and $t_{0}$ for $t_{e}=10 \mu \mathrm{s}$, see Fig. 7 (top). The corresponding simulated effective gains for $t_{e}=10 \mu \mathrm{s}, F=23000$ and a steady state

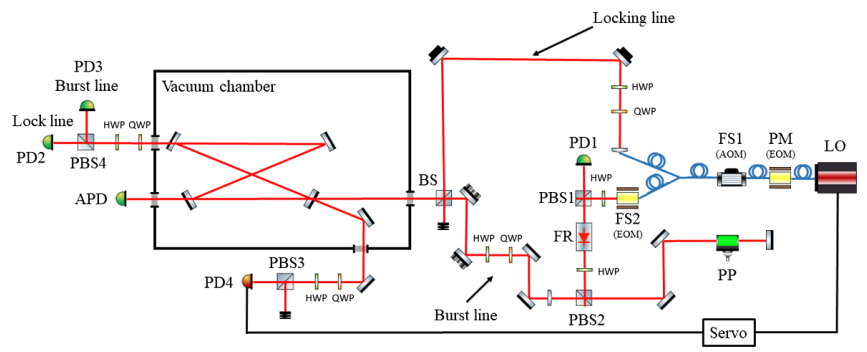

FIG. 5. Setup used for experimental validation of optimization of the burst mode cavity parameters. LO: cw laser oscillator; PM: phase modulator; FS: frequency shifter; EOM: electro-optic modulator; AOM: acousto-optic modulator; PBS: polarizing beam splitter; BS: nonpolarizing beam splitter; FR: Faraday rotator; PP: pulse picker; PD: photodiode; APD: avalanche photodiode; SERVO: Pound-Drever-Hall locking feedback system. gain of 9600 are plotted in the bottom of Fig. 7. Experimental and simulated plots are in fair agreement. Both exhibit a maximum at $t_{p} \simeq 58 \mu \mathrm{s}$ and $t_{0} \simeq 52 \mu \mathrm{s}$. Note also that the absolute scale of the distributions is also in fair agreement, given the best knowledge of the measured cavity transmission parameter $T_{1}=180 \times 10^{-6}$ and the measured finesse of 23000 . The experimental effective gains are also calculated for various values of $t_{e}$ with the arrival time $t_{0}$ optimized using Eq. (11), see Fig. 8 (top). Again, data show good agreement with the simulated plots for $F=23000$ and a steady state gain of 9600, see Fig. 8 (bottom).

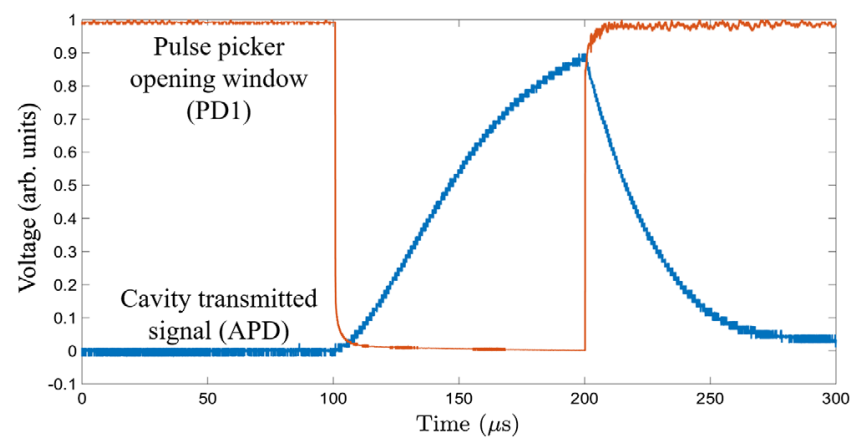

FIG. 6. Example of experimental signal traces (see text for details).
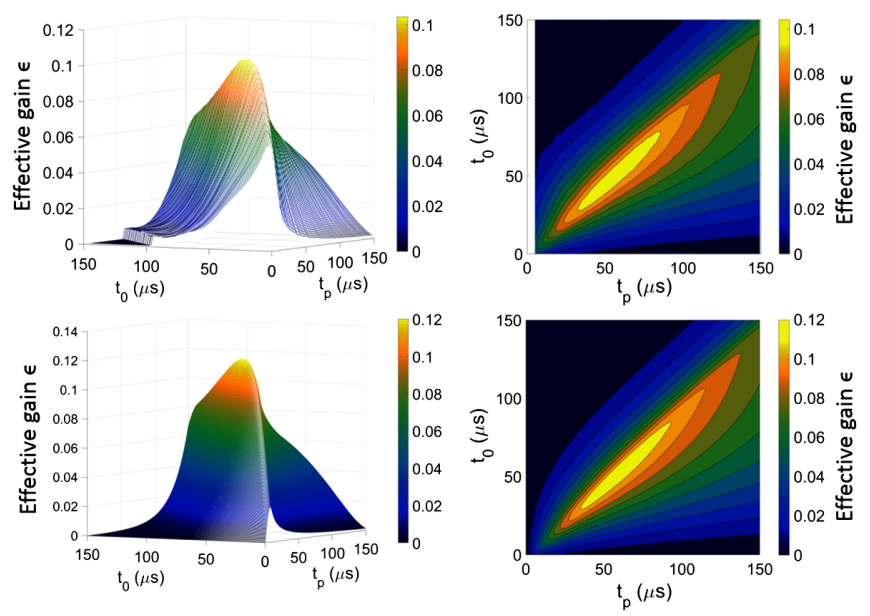

FIG. 7. Top: Experimental effective gains versus $t_{p}$ and $t_{0}$ for $t_{e}=10 \mu \mathrm{s}$. Bottom: Simulated effective gains versus $t_{p}$ and $t_{0}$ for $t_{e}=10 \mu \mathrm{s}, F=23000$ and a steady state gain of 9600 . 

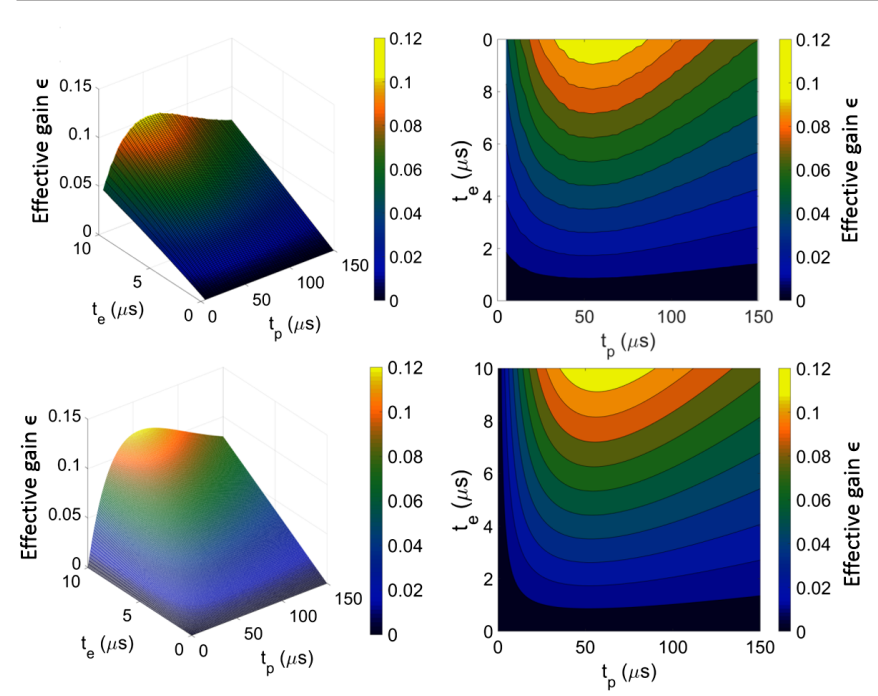

FIG. 8. Top: Experimental effective gains versus $t_{p}$ and $t_{e}$ for optimized $t_{0}$. Bottom: Simulated effective gains versus $t_{p}$ and $t_{e}$ for optimized $t_{0}, F=23000$ and a steady state gain of 9600 .

\section{SUMMARY}

In this article, formal and experimental studies of FPCs operated in burst mode have been conducted in the context of Compton scattering. It is shown that optimum parameter values for the laser system and the optical cavity exist provided that the number of bunches in the electron-beam train and their time separation are known. A good qualitative agreement was obtained between the model and the experimental data. The effective gain $\epsilon$ of the FPC operated in burst mode has been used has the figure of merit for this optimization. A possible upgrade of the STAR Compton source is used as a numerical example. In this example values of the order of 500 are obtained for $\epsilon$, which allows to use low average power (of a few watts) seed laser while the peak power in the FPC is expected to be of a few tens of MW, and so far unprecedented to the best of our knowledge. These results show that such systems may be of interest for future designs of high-flux, high-quality, low cost x-ray facilities based on Compton scattering. It also paves the way towards more advanced studies that would concentrate on an optimization of all machine parameters, not restricted to the optical system.

\section{ACKNOWLEDGMENTS}

The authors warmly thank K. Sakaue for fruitful discussions and information related to his studies of FPCs operated in burst mode.

[1] H. Kogelnik and T. Li, Laser beams and resonators, Appl. Opt. 5, 1550 (1966).

[2] R. J. Jones, K. D. Moll, M. J. Thorpe, and J. Ye, PhaseCoherent Frequency Combs in the Vacuum Ultraviolet via
High-Harmonic Generation inside a Femtosecond Enhancement Cavity, Phys. Rev. Lett. 94, 193201 (2005).

[3] I. Pupeza et al., Compact high-repetition-rate source of coherent $100 \mathrm{eV}$ radiation, Nat. Photonics 7, 608 (2013).

[4] G. Moortgat-Pick et al., Polarized positrons and electrons at the linear collider, Phys. Rep. 460, 131 (2008).

[5] O. Adriani et al., Technical Design Report EuroGammaS proposal for the ELI-NP Gamma beam System, arXiv: 1407.3669.

[6] A. Chaleil, V. Le Flanchec, A. Binet, J. P. Nègre, J. F. Devaux, V. Jacob, M. Millerioux, A. Bayle, P. Balleyguier, and R. Prazeres, Inverse Compton scattering X-ray source yield optimization with a laser path folding system inserted in a pre-existent RF linac, Nucl. Instrum. Methods Phys. Res., Sect. A 840, 113 (2016).

[7] M. Y. Shverdin, I. Jovanovic, V. A. Semenov, S. M. Betts, C. Brown, D. J. Gibson, R. M. Shuttlesworth, F. V. Hartemann, C. W. Siders, and C. P. J. Barty, Opt. Lett. 35, 2224 (2010).

[8] Z. Huang and R. D. Ruth, Laser-Electron Storage Ring, Phys. Rev. Lett. 80, 976 (1998).

[9] J. Bonis et al., Non-planar four-mirror optical cavity for high intensity gamma ray flux production by pulsed laser beam Compton scattering off GeV-electrons, J. Instrum. 7, P01017 (2012).

[10] I. Chaikovska et al., High flux circularly polarized gamma beam factory: coupling a Fabry-Perot optical cavity with an electron storage ring, Sci. Rep. 6, 36569 (2016).

[11] P. Walter, A. Variola, F. Zomer, M. Jaquet, and A. Loulergue, A new high quality X-ray source for Cultural Heritage, C.R. Phys. 10, 676 (2009).

[12] E. Eggl, M. Dierolf, K. Achterhold, C. Jud, B. Günther, E. Braig, B. Gleich, and F. Pfeiffer, The munich compact light source: Initial performance measures, J. Synchrotron Rad. 23, 1137 (2016).

[13] H. Ikeura-Sekiguchi et al., In-line phase-contrast imaging of a biological specimen using a compact laser-Compton scattering-based x-ray source, Appl. Phys. Lett. 92, 131107 (2008).

[14] T. Akagi et al., Narrow-band photon beam via laser Compton scattering in an energy recovery linac, Phys. Rev. Accel. Beams 19, 114701 (2016).

[15] A. Ando et al., Isochronous storage ring of the New SUBARU project, J. Synchrotron Radiat. 5, 342 (1998).

[16] S. Amano, K. Horikawa, K. Ishihara, S. Miyamoto, T. Hayakawa, T. Shizuma, and T. Mochizuki, Several-MeV $\gamma$-ray generation at NewSUBARU by laser Compton backscattering, Nucl. Instrum. Methods Phys. Res., Sect. A 602, 337 (2009).

[17] H. R. Weller, M. W. Ahmed, H. Gao, W. Tornow, Y. K. Wu, M. Gai, and R. Miskimen, Research opportunities at the upgraded HI $\gamma S$ facility, Prog. Part. Nucl. Phys. 62, 257 (2009).

[18] Y. Du et al., Generation of first hard X-ray pulse at Tsinghua Thomson Scattering X-ray Source, Rev. Sci. Instrum. 84, 053301 (2013).

[19] K. Sakaue, M. Washio, S. Araki, M. Fukuda, Y. Higashi, Y. Honda, T. Omori, T. Taniguchi, N. Terunuma, J. Urakawa, and N. Sasao, Observation of pulsed X-ray trains produced 
by laser-electron Compton scatterings, Rev. Sci. Instrum. 80, 123304 (2009).

[20] K. Sakaue et al., in Proceedings of the 22nd Particle Accelerator Conference, PAC-2007, Albuquerque, NM (IEEE, New York, 2007), pp. 1034-1036, DOI: 10.1109/ PAC.2007.4440973.

[21] K. Sakaue, S. Araki, M. Fukuda, Y. Higashi, Y. Honda, N. Sasao, H. Shimizu, T. Taniguchi, J. Urakawa, and M. Washio, Development of a laser pulse storage technique in an optical super-cavity for a compact X-ray source based on laser-Compton scattering, Nucl. Instrum. Methods Phys. Res., Sect. A 637, S107 (2011).

[22] K. Sakaue, M. Washio, S. Araki, M. Fukuda, Y. Honda, N. Terunuma, and J. Urakawa, Stabilization of burst laser pulse storage in an optical enhancement cavity using a counter propagating mode, Rev. Sci. Instrum. 89, 023305 (2018).

[23] A. Rakhman and Y. Liu, in High-Brightness Sources and Light-Driven Interactions (Optical Society of America, California, USA, 2016), p. JM7A.9, DOI: 10.1364/EUVXRAY.2016.JM7A.9.

[24] S. Breitkopf et al., A concept for multiterawatt fibre lasers based on coherent pulse stacking in passive cavities, Light Sci. Appl. 3, e211 (2014).

[25] T. Zhou, J. Ruppe, C. Zhu, I-Ning Hu, J. Nees, and A. Galvanauskas, Coherent pulse stacking amplification using low-finesse Gires-Tournois interferometers, Opt. Express 23, 7442 (2015).

[26] H. Carstens et al., Megawatt-scale average-power ultrashort pulses in an enhancement cavity, Opt. Lett. 39, 2595 (2014).

[27] L. Gallais and M. Commandré, Laser-induced damage thresholds of bulk and coating optical materials at 1030 nm, 500 fs, Appl. Opt. 53, A186 (2014).

[28] A. Deshpande, S. Araki, M. Fukuda, K. Sakaue, N. Terunuma, J. Urakawa, and M. Washio, Experimental results of an rf gun and the generation of a multibunch beam, Phys. Rev. ST Accel. Beams 14, 063501 (2011).

[29] L. Piersanti et al., in Proceedings of the International Particle Accelerator Conference (IPAC16), Busan, Korea, 2016, International Particle Accelerator Conference No. 7 (JACoW, Geneva, Switzerland, 2016), pp. 407-410.

[30] T. Suzuki, General formulae of luminosity for various types of colliding beam, Report No. KEK-76-3, 1976.

[31] A. L. Bullington, B. T. Lantz, M. M. Fejer, and R. L. Byer, Modal frequency degeneracy in thermally loaded optical resonators, Appl. Opt. 47, 2840 (2008).
[32] C. Baumgarten, M. Pedicone, H. Bravo, H. Wang, L. Yin, C. S. Menoni, J. J. Rocca, and B. A. Reagan, $1 \mathrm{~J}, 0.5 \mathrm{kHz}$ repetition rate picosecond laser, Opt. Lett. 41, 3339 (2016).

[33] M. Schulz, R. Riedel, A. Willner, S. Düsterer, M. J. Prandolini, J. Feldhaus, B. Faatz, J. Rossbach, M. Drescher, and F. Tavella, Pulsed operation of a high average power Yb:YAG thin-disk multipass amplifier, Opt. Express 20, 5038 (2012).

[34] G. Rempe, R. J. Thompson, H. J. Kimble, and R. Lalezari, Measurement of ultralow losses in an optical interferometer, Opt. Lett. 17, 363 (1992).

[35] M. J. Lawrence, B. Willke, M. E. Husman, E. K. Gustafson, and R. L. Byer, Dynamic response of a Fabry-Perot interferometer, J. Opt. Soc. Am. B 16, 523 (1999).

[36] A. Bacci et al., in Proceedings of the 5th International Particle Accelerator Conference (IPAC 2014): Dresden, Germany, 2014 (JACoW, Geneva, Switzerland, 2014), WEPRO115, pp. 2238-2241, DOI: 10.18429/JACoWIPAC2014-WEPRO115.

[37] A. Bacci et al., in Proceedings of the 7th International Particle Accelerator Conference (IPAC 2016): Busan, Korea, 2016 (2016), TUPOW004, pp. 1747-1750, DOI: $10.18429 /$ JACoW-IPAC2016-TUPOW004.

[38] A. E. Siegman, Lasers (University Science Books, Sausalito, California, USA, 1986).

[39] I. Will, H. I. Templin, S. Schreiber, and W. Sandner, Photoinjector drive laser of the FLASH FEL, Opt. Express 19, 23770 (2011).

[40] E. I. Gacheva, V. V. Zelenogorskii, A. V. Andrianov, M. Krasilnikov, M. A. Martyanov, S. Y. Mironov, A. K. Potemkin, E. M. Syresin, F. Stephan, and E. A. Khazanov, Disk Yb:KGW amplifier of profiled pulses of laser driver for electron photoinjector, Opt. Express 23, 9627 (2015).

[41] H. Braun et al., in Proceedings of the 19th Particle Accelerator Conference, Chicago, IL, 2001 (IEEE, Piscataway, NJ, 2001), p. 70, DOI: 10.1109/PAC.2001.987614.

[42] D. Sertore et al., in Proceedings of the 21st Particle Accelerator Conference, Knoxville, 2005 (IEEE, Piscataway, NJ, 2005). p. 671.

[43] R. W. P. Drever, J. L. Hall, F. V. Kowalski, J. Hough, G. M. Ford, A. J. Munley, and H. Ward, Laser phase and frequency stabilization using an optical resonator, Appl. Phys. B 31, 97 (1983).

[44] A. Rakhman, M. Notcutt, and Y. Liu, Power enhancement of burst-mode ultraviolet pulses using a doubly resonant optical cavity, Opt. Lett. 40, 5562 (2015).

[45] X. Liu et al., Laser frequency stabilization using folded cavity and mirror reflectivity tuning, Opt. Commun. 369, 84 (2016). 